\title{
Translanguaging and multilingual academic literacies:

\author{
How do we translate these into French? \\ Pour en faire quoi ? (et pourquoi s'en faire?)
}

\author{
Guillaume Gentil \\ guillaume.gentil@carleton.ca \\ CARLETON UNIVERSITY
}

\begin{abstract}
Translanguaging has become an inescapable notion in the anglophone literature on multilingual education. Questions thus arise as to whether and how it should be imported into francophone educational discourse, which has developed parallel vocabularies and orientations. The stakes are not only terminological - how to import a notion with ill-defined contours from one discursive universe to another - but pedagogical and political: what value could be added by the notion of translanguaging, either translated or borrowed as is, not only in addressing the needs of plurilingual writers who, like francophone educators, must reconceptualize English disciplinary discourses in another language, but also in creating more equitable conditions for the circulation of ideas across linguistic and national lines? To tackle these questions, this paper draws on two sources of insight: strategies for cross-lingual mediation developed in translation and terminology studies, and lessons from the import of literacy into francophone discourse in the 1990s and 2000s. Existing uses and translations of translanguaging are then reviewed, and new translation equivalents proposed to render and clarify the multiple meanings of translanguaging and operationalize the notion in French. In keeping with a translanguaging approach, the paper translanguages about translanguaging, deepening our understanding of it through more than one linguistic lens.
\end{abstract}

Key words: translanguaging, translation and writing pedagogy, multilingual terminology, multilingual academic literacies, crosslingual reading and composing, international circulation of ideas

\section{Résumé}

Translanguaging est devenu une notion incontournable de la littérature anglophone sur l'éducation multilingue. La question se pose donc de savoir dans quelle mesure et comment l'importer dans le discours éducatif francophone, lequel a développé des vocabulaires et des orientations 
parallèles. L'enjeu n'est pas seulement terminologique — comment importer une notion aux contours plus ou moins bien définies d'un univers discursif à un autre - mais aussi pédagogique et politique : quelle valeur ajoutée cette notion, traduite ou empruntée telle quelle, pourrait-elle apporter, non seulement pour répondre aux besoins des scripteurs plurilingues qui, comme les éducateurs francophones, doivent reconceptualiser les discours disciplinaires anglais dans une autre langue, mais aussi pour créer des conditions plus équitables de la circulation des idées pardelà les frontières linguistiques et nationales ? Pour répondre à ces questions, cet article puise son inspiration de deux sources : les stratégies de médiation interlinguistique élaborées en traductologie et en terminologie, et les leçons tirées de l'importation de literacy en français dans les années 1990 et 2000. Les utilisations et les traductions existantes de translanguaging sont ensuite examinées, et de nouveaux équivalents proposés, selon les multiples acceptions du terme, pour rendre la notion opératoire en français tout en la clarifiant. Dans la logique d'une démarche translangagière, ce texte translangage sur le translangageage afin de mieux le comprendre à travers un prisme plurilingue.

Mots-clés : orientation translangagière, traduction et didactique de l'écrit, terminologie multilingue, littératies universitaires plurilingues, alternance des langues en lecture et en écriture, circulation internationale des idées

\section{Problématique - At issue}

(1) One of the main difficulties of translanguaging is conceptual and terminological ... The difficulty of using the same term for different phenomena is related to the attractiveness of the term ... (Cenoz \& Gorter, 2017, p. 309)

(2) Il y a plus de termes exacts en anglais qu'en français ... Juste le terme « gender studies. » On dirait qu'en anglais ça veut dire quelque chose, mais en français, «études sur les genres, » c'est comme ... tu sais le concept n'est pas autant connu. Je trouve qu'en anglais, il y a plus de termes qui vont exprimer précisément une idée. (Excerpt from an interview with Katia, a Frenchspeaking doctoral student from Québec, June 30, 2000)

(3) Translanguaging as a pedagogical practice not only promotes a deeper understanding of content, but also develops the weaker language in relationship with the one that is more dominant. (Wei \& García, 2017, p. 229)

Since its unassuming introduction from Welsh in the early 2000s (Lewis, Jones, \& Baker, 2012), translanguaging has become an inescapable concept in the English literature on bi- and multilingual education. Such fast-paced ascent can be seen in library databases (259 records in the Linguistics and Language Behavior Abstracts database since 2010 on August 15, 2018, more than half of which since 2016) and the central place it occupies in the third edition of the Encyclopedia of Language and Education (Cenoz, Gorter, \& May, 2017). 
In the beginning, the term was restricted to an innovative language allocation strategy in Welsh bilingual programs, alternating input and output in two languages across lessons to promote content learning and literacy development in and across two languages (Baker, 2003). As it became popular, translanguaging has gained a constellation of new meanings, referring, among other things, to instructional strategies, language practices, and theoretical orientations in bilingual education, which has led to some difficulty in pinpointing exactly what it may mean, for whom, and in what context. As Cenoz and Gorter astutely remark in the first opening quote, the very fluidity of the term translanguaging itself has contributed to both its attractiveness and its fuzziness.

In contrast, to my knowledge, translanguaging has not made major inroads into the Francophone literature on bi- or plurilingualism and language learning in multilingual ${ }^{1}$ contexts, which has developed both in relation and in parallel to its Anglophone counterpart. In introducing a special issue of the International Journal of Multilingualism on "French voices on plurilingualism and pluriculturalism," Moore and Gajo (2009) offer an overview in English for English audiences of key constructs and lines of thinking that have been developed in (mostly European) Francophone scholarship. Chief among these is the concept of plurilingual and pluricultural competence (compétence plurilingue et pluriculturelle), which, not unlike translanguaging, has been construed as signalling an ideological shift in bilingualism and language education away from monolingualist perspectives and toward more open, flexible, holistic, dynamic, and socially situated conceptions of language learning and language teaching in contexts of language contact and linguistic diversity (Castellotti \& Moore, 2015).

Some translanguaging scholars have questioned the common view that named languages such as English and French constitute autonomous language

\footnotetext{
${ }^{1}$ Distinctions have been made between multilingue et plurilingue in French, and multilingual and plurilingual in English. In this text, I distinguish between multi- and pluri- first on etymological grounds, with multi- referring to "many" and pluri- to "several." Arguably, the distinction between "many" and "several" is not always clear or relevant, and so multi- and pluri- can sometimes be used interchangeably. However, to me these prefixes also seem to evoke difference discourses on linguistic diversity. I thus use plurilingue/plurilingual when I refer specifically to the concept of plurilingual and pluricultural competence or when I want to evoke, more loosely, European and Francophone discourses on plurilingualism, which I see as advocating linguistic pluralism, that is, competence in and the use of several languages by institutions and individuals. I use multilingue/multilingual when referring to an undefined and potentially unlimited number of languages (as seems to be the case in some Anglophone discourses on linguistic diversity) or, as in some versions of translanguaging, when languages cannot be separated and thus it does not make sense to count them.
} 
systems in individual minds, arguing instead that they constitute "one linguistic repertoire with features that have been socially constructed as belonging to separate languages" (García \& Wei, 2014, p. 2; see also Otheguy, García, $\&$ Reid, 2015). The effect of the social construction of language as languages on bilinguals' mental representations and cognitive processing is a fascinating yet largely unresolved question (but see MacSwan, 2017; Pavlenko, 2005). Without entering into this complex issue, and acknowledging the detrimental effects that insisting on monolingual norms can have on multilinguals in school and society, one should not lose sight of some of the more positive aspects of languages being constructed as separate systems. To use M.A.K. Halliday's (1978) apt metaphor, a linguistic system such as the English language represents a "shared meaning potential" comprising all of what its users can mean (p. 1). Different linguistic systems or traditions like English and French bring with them their own sets of resources for meaning-making, reflecting their histories and the environments in which they evolved and privileging certain ways of attending to the world and one's experience in it. Indeed, one belief underlying translanguaging as an approach to bilingual education, in the original sense of alternating languages for teaching and learning, is that making sense of a subject matter through two (or more) languages helps to gain deeper, novel understandings of it (Lewis et al., 2012).

A similar argument about the value of language alternation, which supposes some degree of language separation, could be extended from bilingual education to multilingual scholarship. The point of shuttling between academic discourses, understood as having come into being through different linguistic traditions, is that different languages may afford unique concepts, metaphors, ways of thinking and noticing, and thus bring complementary contributions to the construction of knowledge. Translanguaging is a case in point. The coinage appears to have opened new lines of inquiry in English scholarship. What affordances could it bring to French scholars and language educators? Should it be borrowed as is, as is commonly done in the few occurrences I have found in the French-medium literature, and as the organizers of the CCERBAL 2018 conference have done? Should we look for equivalent notions that have been expressed through different means, evoking different discourses, images or metaphors, in French? Or should we draw on French linguistic resources to propose a neologism with similar meanings?

\section{Enjeu pédagogique - Pedagogical challenge}

At the root of these questions lies not only a theoretical or terminological problem, but also a pedagogical and political one. My interest in the concept of translanguaging originates in my research on the academic biliteracy practices of Francophone students and scholars, who appeared to be struggling to 
tackle in French notions and arguments they have encountered in the Englishmedium literature of their field of study when these notions and arguments have not been named, framed, or conceptualized in similar ways in francophone discourses (Gentil, 2005). Such struggles were particularly salient in the case of Katia, a francophone doctoral student at an English-speaking university in Quebec, who had to write a literature review on gender studies in French. The task was challenging, not only because the literature she was drawing on was almost exclusively in English, but also because the concept she was writing about — gender — did not have well-established equivalents in French (on this, see Raus, 2013). She rightly intuits in the second opening vignette that, while "gender studies" evokes a well-known field of study in English, the ontological status of "études du genre" in the Francophone world is not as clear. That was particularly true at the time she was writing, in the early 2000s, even though gender studies owe much to the writings of Simone de Beauvoir and other French feminist thinkers (Parini, 2010).

Katia's pains resonated with my own experience, as a francophone doctoral student of second language education struggling to write bilingually about academic biliteracy, at a time when the very concept of literacy, let alone biliteracy, was foreign to French vocabulary. I also came to realize that plurilingual university writers were ill-prepared for writing in one language from sources in another and mediating between discourse communities using different languages, in large part because writing instruction was compartmentalized along language lines (L1 or L2, English or French) and sequestered from translation training. It is in this context that my discovery of translanguaging, in Baker (2001, 2003), was epiphanic: not only did the term capture the alternation of languages in reception and production that I was trying to describe; it also suggested a pedagogical avenue for preparing plurilingual writers for this type of linguistic juggling. It went against the sacrosanct principle of language separation in French immersion. If such language alternation strategies had been used in the Welsh bilingual education context, could they also be considered in the Canadian and French contexts I was familiar with? Had they been tried in Francophone settings? How had they been named, or how could they be named?

My encounter with an English word, translanguaging, and my attempt to work with it through English and in French - in essence, to translanguage about translanguaging - illustrates how the circulation of ideas across linguistic and national lines can generate new lines of thought. Of particular interest are these questions: what are the pedagogical affordances of translanguaging as a concept for thinking about plurilingual writers' language needs and ways to better address them? How could these affordances be best rendered in French and exploited in French-speaking contexts? 


\section{Enjeu politique - Political ramifications}

Questions about terminological equivalents may appear trivial — why not just borrow translanguaging as is into French and get on with it? They might well be, were they not arising against a backdrop of power struggles over the control of discursive spaces and economic resources. In the opening vignette, Katia expressed doubt less about her own linguistic abilities and more about the capacity of the French language itself as a suitable medium of scholarship. Her mother tongue, which seems essential to her self-definition as her language of inheritance and affiliation, does not, she feels, have as many exact terms to precisely express ideas as English. Francophones' propensity to lose confidence in French as a language of scholarship emerged with surprising regularity from the testimonials I gathered in France and in Québec:

En français, t'as pas les mots donc tu tournes autour du pot. En tout cas, dans le domaine scientifique, c'est flagrant.

Je pense que c'est plus facile de créer des termes en anglais. C'est une langue beaucoup plus imagée, qu'en français, [qui] est une langue beaucoup plus rigide. (Gentil, 2002, p. 166)

Taking these testimonials at face value, it might be tempting to view translanguaging as yet another example of the greater expressive abilities of the English language and borrow the term to compensate for a perceived lack of precise or vivid equivalent terminology in French. Alternatively, however, one could interrogate the unattended influences and unintended consequences of Francophone scholars' propensities to devalue their own language as a suitable medium for research and higher education. The greater the pressure to publish in English, the more knowledge is constructed in this language. It is not surprising, then, that disciplinary discourses and associated vocabularies tend to be developed in English first and then circulated in other languages; but the more other languages seem to be falling behind as they are trying to catch up, the less attractive they become, and the more English becomes hegemonic (cf. Ammon, 1996). That Francophone scholars (like other non-Anglophone scholars, cf. Lillis \& Curry, 2010) find themselves composing in their own language from English sources, presumably more frequently than Anglophone scholars find themselves composing in English from non-English sources, thus reflects, and reproduces, the power differential between English and French (and other languages) as languages for the construction and dissemination of knowledge. It reflects the competing pressures for Francophone scholars to read and publish in English and yet to continue to use French to reach out to Francophone audiences, for popularization purposes and out of linguistic loyalty (e.g., Gentil \& Séror, 2014). 
Questions about whether, how, and why Francophone scholars and language educators should adopt a concept such as translanguaging into their academic discourses and educational practices are thus not solely terminological. They arise within a larger backdrop. As scholars adopt a terminology, they align themselves with certain discourses and perspectives, and contribute to the visibility, or symbolic capital (cf. Bourdieu, 1994), of those who coined it or are associated with it. This is especially true in the social sciences, where academic vocabularies tend to be less standardized and thus more open for competition than in the physical sciences. Parini (2010), for example, traced how the adoption and translation of "gender" from English into French academic discourse was part of broader epistemological, institutional, and political shifts in France's academia and identity politics. What are, then, the broader epistemological, discursive, and political stakes behind the possible adoption and rendering of translanguaging into French academic discourse?

\section{Let's translanguage on translanguaging - Translangageons sur le translangageage !}

The very topic of this paper inevitably raised the question of the language in which it would be preferable to write it. On the one hand, I suspect that the questions raised are likely to be of primary interest to Francophone and Francophile readers who use French in their professional practice. On the other hand, I am hoping to capture the interest of other potential audiences who may not (comfortably) read academic French and yet entertain similar questions about the international circulation of concepts across linguistic lines and the preservation of non-Anglophone discursive academic spaces. For example, there might be parallels to be drawn between questions about the import of translanguaging into French described here and similar questions vis-à-vis other languages such as Spanish, Chinese, or German. I am grateful for the opportunity given by the editors of this special issue to mix and alternate languages. To my knowledge, such a linguistic strategy is rare and innovative in language publishing, and yet quite apt given the topic of the special issue.

The third opening vignette draws attention to Wei and García's (2017) observation that translanguaging "not only promotes a deeper understanding of content, but also develops the weaker language in relationship with the one that is more dominant" (p. 229). Indeed, this seems to be a finding that emerged from research on translanguaging in the Welsh context of education: students appear to develop a deeper understanding of a subject matter while studying it bilingually; at the same time, they develop a stronger command of Welsh, and the Welsh language gains in status, use, and vocabulary (Lewis et al., 2012). As observed earlier, the increasing popularity of translanguaging, at least as a notion if not an educational practice, has made it harder to grasp exactly what 
the term can mean. I would hope that thinking through the term in and across English and French, that is, translanguaging about "translanguaging," will help unpack the various meanings it has come to acquire, as French equivalents are likely to differ for each meaning. And I would hope that such an exercise will also contribute to developing French terminology, and enhance the positioning of French academic discourses vis-à-vis their English counterparts.

Alternating languages in a paper does raise certain logistical difficulties, not least in achieving a certain balance between the languages and yet continuing to engage audiences who may not understand one language well or at all. One strategy might be, as in federal Canadian signage or communication with the public, to aim for a perfect symmetry of treatment with equal textual space being devoted to English and French. However, this would ignore the imbalance between the two languages that I just described. In a pedagogical situation, it would probably be advisable to safeguard more space (and instructional time) for the minoritized language. Unfortunately, I do not have the luxury in this paper to devote much space to scaffold reading comprehension and language development. Nor is it the primary purpose of an academic communication. Like the plenary session on which this written paper is based, I thus find it preferable to skew the paper in English at the expense of French on the (perhaps wrong) assumption that more of the target readership will be able to read English than French. That is why I felt it important to introduce and conclude this paper in English. However, two of the middle sections are written in French. I hope that they will be intelligible to most readers. However, for those who cannot read French, I hope that this will be helpful:

- Whenever possible, I have included references to other publications in English where I developed similar ideas, although from a different angle and with varying degrees of detail.

- I have translated headings and provided parenthetical translations of key terms.

- Copying and pasting text into the DeepL Translator, ${ }^{2}$ arguably better than Google Translate for English and French, should help you follow the gist of the argument. It may also help you appreciate the strengths and limitations of these online translation tools, which are becoming more performant for gisting (i.e., to understand the gist of a text in an unknown language) but are still far from adequate for bilingual composition. They may thus be worth considering as an option for receptive multilingualism in publishing where authors compose in their stronger language and readers use online translation into their preferred language.

\footnotetext{
${ }^{2}$ DeepL Translator: https://www.deepl.com/en/translator
} 
I have also chosen to compose the middle section, on the import of literacy into French, in English, as most of the references cited are in French; conversely, the section, in French, about the various meanings that translanguaging has gained in the English literature, draws on mostly English sources. I thus hope to help mediate between the Francophone and Anglophone discursive universes, brokering readers into literatures they may not be aware of. That said, despite these efforts at making my argument intelligible to the broadest readership possible, I recognize that it is readers who are conversant in both English and French are likely to make the most of this paper. As Creese and Blackledge (2010) point out, in translanguaging, "Both languages are needed simultaneously to convey the information ... each language is used to convey a different informational message, but it is in the bilingualism of the text that the full message is conveyed" (p. 108).

Composing in one language from sources in another, navigating across linguistic and discursive communities, and introducing a new concept such as translanguaging from English to French academic discourses have this in common: they require good skills in translation. In the next section, I turn to what translation and terminology studies may have to offer, not only to plurilingual scholars in search of terminological equivalents, but also, and more broadly, to language educators eager to develop a plurilingual approach to writing instruction that equips university students and scholars for professional and academic communication in a global world. While the interests of language educators and translation specialists diverged in the latter half of the 20th century, commonalities in professional translators' and plurilingual writers' translanguaging needs may call for a rapprochement (cf. Cook, 2010; Gentil, 2018). A second avenue I then explore in investigating the import of translanguaging into French is provided by lessons to be drawn from an earlier import, in the 1990s and early 2000s: that of "literacy" and derivatives such as "biliteracy" and "computer literacy." Resistance to such imports at the time has noteworthy parallels to questions that can now be raised about translanguaging, so it would be interesting to see how littératie (or littéracie) gained a foothold in Francophone education discourse and what added value it may have brought in terms of epistemological and disciplinary shifts. Such an exploration will be even more helpful because, from the very beginning, the concept of translanguaging has been defined in its relations to "biliteracy," "dual literacy," and "transliteracy" (Baker, 2003; Lewis et al., 2012). As "translanguaging" gained popularity in the Anglophone educational literature, it was biliteracy's turn to be redefined, and repositioned, vis-à-vis translanguaging (Hornberger \& Link, 2012). In light of the insights gained from translation studies and the import of "literacy," I then examine how "translanguaging" has been used or translated in the Francophone literature, drawing parallels with similar notions that seem 
to have appeared independently in French, and offering possible translation equivalents for the various meanings of translanguaging that I recorded in the Anglophone literature. I conclude this intellectual journey by suggesting the possible added value that importing the notion of translanguaging, in some or all of its denotational complexity and ideological resonance, into French educational discourse might bring for understanding plurilingual writers' composing practices and academic literacy development, and redrawing disciplinary and institutional lines in ways that help design and implement plurilingual approaches to writing instruction.

\section{How translation and terminology studies might help- L'apport des traductologues et des terminologues}

Il est intéressant de constater comment la formation des traducteurs et la didactique des langues semblent avoir eu le besoin de s'émanciper l'une de l'autre. Avant l'émergence de la traductologie en tant que champ d'études autonome (sous le vocable translation studies en anglais), dans les années 1970 et 1980, les méthodes d'enseignement de la traduction s'alignaient sur celles de l'apprentissage des langues étrangères. La compétence en traduction étant ainsi conçue essentiellement comme reposant sur une bonne connaissance des langues de travail, la formation des traducteurs insistait sur le perfectionnement linguistique par la compréhension des textes sources et leur réexpression dans la langue cible (Guidère, 2016, pp. 117-118). D'un autre côté, la pratique d'exercices de traduction avait la part belle dans les cours de langues (Cook, 2010). Parallèlement à l'autonomisation de la traductologie, les approches utilisées en formation de traducteurs se sont diversifiées (Guidère, 2016), alors que la traduction devenait l'emblème d'une pédagogie ringarde eu égard aux approches prônées en didactique des langues, plus ou moins éphémères mais excluant toutes, en théorie du moins, le recours à la langue maternelle (Cook, 2010). L'absence, voire le rejet, de la traduction se reflète en particulier dans les orientations des programmes d'anglais sur objectifs universitaires (English for Academic Purposes), ainsi que dans les autres formes de soutien à l'écrit (Centres d'aide à la rédaction/Writing Centres, cours de rédaction universitaire, technique ou professionnelle en langue première). Au cloisonnement de l'enseignement de la rédaction par langues (anglais ou français, langue première ou langue seconde) s'ajoute l'autonomisation de l'enseignement de la traduction, dans des unités et des programmes distincts, réservés aux futurs professionnels de la traduction (Gentil, 2006).

Cette évolution explique sans doute pourquoi les scripteurs plurilingues, à l'université du moins, semblent très peu sensibilisés aux stratégies enseignées dans les programmes de formation de traducteurs bien qu'elles puissent leur être très utiles (cf. Gentil, 2019). Si la traductologie a pu élaborer des 
ressources qui lui sont propres grâce à une disciplinarisation émancipatrice, l'heure serait peut-être venue d'envisager les rapports entre traductologie, rédactologie et didactique des langues dans une optique d'enrichissement mutuel. Les appels à réhabiliter la traduction dans l'enseignement des langues (e.g., Cook, 2010; Gentil, 2018; Göpferich, 2017) pourraient bien jeter les bases de tels rapprochements.

Bien qu'ils ne se recoupent pas entièrement, les besoins des traducteurs et des scripteurs plurilingues semblent en effet en partie communs. C'est le cas notamment au niveau du travail de réexpression d'une langue à l'autre, dont la nécessité de trouver des équivalents terminologiques et phraséologiques. Les scripteurs ont peut-être l'avantage de mieux connaître le domaine dans lesquels ils écrivent, mais les traducteurs sont plus au fait des méthodes en terminologie multilingue. La principale différence communément admise entre traduction et rédaction est que le traducteur travaille essentiellement sur la forme, en partant d'un texte déjà donné, tandis que le rédacteur doit composer un texte, soit travailler à la fois sur le fond et sur la forme. La posture d'auteur confère a priori une plus grande maîtrise, ou autorité, sur le texte, que celle de traducteur, qui impose souvent un rapport de déférence envers le texte source. Le traducteur, un peu à l'instar du réviseur linguistique, reste ainsi dans l'ombre de l'auteur, ne bénéficiant pas de la même reconnaissance ou notoriété. Cette conception de la traduction explique sans doute pourquoi il peut s'avérer difficile de motiver des étudiants en traduction pour un enseignement de la rédaction n'ayant pas pour seul objectif le perfectionnement d'un code linguistique, bien que cet enseignement puisse s'avérer bénéfique (Kruse, 2012; Schrijver, van Vaerenbergh, Leijten et Van Waes, 2014). Il est généralement aussi entendu que les traducteurs travaillent vers leur langue dominante, tandis que les scripteurs plurilingues peuvent être amenés à rédiger dans des langues qu'ils maîtrisent plus ou moins bien. Les pratiques de travail sont pourtant plus complexes que cette vision traditionnelle de la répartition des tâches. En effet, les scripteurs plurilingues en contexte universitaire composent le plus souvent leurs textes à partir de sources, qu'ils doivent citer, résumer ou paraphraser pour situer leur propre discours dans le contexte discursif de leur domaine. Rechercher de l'information dans plusieurs langues et la synthétiser d'une langue à l'autre semble donc bien être un travail commun des traducteurs professionnels et des scripteurs universitaires en contexte plurilingue (cf. notamment Guidère, 2016, pp. 133-136, sur le travail de veille multilingue des traducteurs). Traducteurs et scripteurs plurilingues partagent en cela une fonction de courtier linguistique et de médiateur interculturel.

Pour se préparer à ce travail de médiation interlinguistique et interculturel, les étudiants en traduction reçoivent une initiation à une panoplie d'outils et de stratégies qui pourraient s'avérer fort utiles aux scripteurs plurilingues. 
En particulier, les travaux en traductique et en terminologie multilingue ont connu un essor important ces vingt dernières années, au Canada comme en Europe, à la suite des développements d'outils informatiques facilitant la compilation et la recherche de bases de données et de corpus de textes (cf., par exemple, L'Homme, 2008; Loock, 2016; Raus, 2013). Figurent en premier lieu les banques terminologiques multilingues, telles que TERMIUM (Canada, n.d.) et Le grand dictionnaire terminologique (Quebec, 2012), qui répertorient des équivalents terminologiques par domaines, pourvus de définitions, d'exemples d'emploi et de commentaires évaluatifs. Mentionnons aussi les concordanciers bilingues comme Linguee ou TradooIT $^{3}$, qui permettent d'extraire des traductions non seulement de termes mais aussi de séquences de plusieurs mots en contexte, à partir de textes bilingues alignés (bitextes) montrant en parallèle des paragraphes de textes traduits d'une langue à l'autre. S'ajoutent à cela les traducteurs en ligne (Google Translate, DeepL), mentionnés précédemment. Bien sûr, ces outils ont chacun leurs points forts et leurs faiblesses (cf. L'Homme, 2008; Loock, 2016; Raus, 2013), d'où une certaine complémentarité, à laquelle les scripteurs plurilingues doivent être sensibilisés au même titre que les étudiants en traduction.

En plus de proposer des outils informatiques d'aide à la traduction de plus en plus performants, la traductologie a aussi élaboré un ensemble d'approches, de modèles et de théories de la traduction qui permettent aux praticiens de faire des choix éclairés en prenant conscience des options qui s'offrent à eux (cf. parmi d'autres, Delisle, 2013; Guidère, 2016; Venuti, 2000). Par exemple, la question se pose souvent de savoir dans quelle mesure une traduction se doit d'être fidèle à l'original. Or il existe toute une réflexion à ce sujet, depuis l'antiquité, oscillant entre deux extrêmes : la fidélité littéraliste et l'adaptation libre, selon les époques et le contexte, avec, en corolaire, une tendance à l'exotisation ou à la naturalisation, selon que les traits étrangers à la culture cible sont gardés ou éliminés lors de la traduction (Guidère, 2016). On sait de plus que les orientations privilégiées reflètent le rapport de force entre cultures sources et cultures cibles : ce n'est pas un hasard si le siècle de Louis XIV est connu pour être «l'âge d'or d'un type de traduction ... baptisé la 'belle infidèle' » (Ballard, 1992, cité par Guidère, 2016, p. 86), les traducteurs de l'époque devant avant tout satisfaire à l'esthétique française en vogue.

Les manuels de traduction proposent différents schémas d'analyses pour sensibiliser les apprentis traducteurs aux choix qu'ils peuvent faire. Pionniers en la matière, Vinay et Darbelnet (1958) ont proposé, dès les années 1950, pour le français et l'anglais, de distinguer entre les procédés « directs » (emprunt, calque, traduction littérale) des procédés « obliques » (transposition, modula-

\footnotetext{
${ }^{3}$ Linguee : www.linguee.com/english-german/about\#about ; TradooIT : www.tradooit.com/.
} 
tion, adaptation). Ces procédés ont fait l'objet de critiques et de réinterprétations depuis, mais ils demeurent utiles pour les scripteurs plurilingues de savoir qu'il est courant, en traduction, de transposer une structure verbale en une structure nominale (transposition : do not enter pour « accès interdit» sur un panneau), de changer de point de vue (modulation : « danger de mort» pour life-threatening danger), ou d'utiliser des moyens linguistiques entièrement différents pour tenter de créer un effet similaire (par ex., dans la traduction d'expressions idiomatiques ou de jeux de mots). Il peut être utile aussi de savoir que traduire nécessite parfois un «étoffement, » c'est-à-dire un ajout de mots par rapport à l'original, ou au contraire un « effacement» (« l'inspecteur chargé de l'enquête » pour the inspector on the case, Vinay et Darbelnet, 1958, p. 111); qu'il faut parfois rendre explicite dans la langue cible un élément sousentendu dans le texte original (explicitation), ou le contraire (implicitation), selon que le sens est inférable ou non à partir du contexte.

Sensibiliser les scripteurs universitaires plurilingues à une gamme d'options en traduction devrait les aider à envisager des choix plus stratégiques et moins mécaniques, en invitant à une plus grande souplesse d'expression sans un recours quasi-exclusif à l'emprunt ou à la traduction littérale, lorsqu'ils cherchent à reformuler en français les concepts et les arguments qu'ils rencontrent en anglais. Pour revenir sur le translanguaging, même s'il est bien sûr possible et peut-être justifié d'emprunter le néologisme tel quel, quelles alternatives à l'emprunt seraient-elles à considérer à la lumière des enseignements de la traductologie et de la terminologie multilingue? Nous reviendrons sur cette question après un examen de l'introduction de la notion de literacy dans les discours francophones, riche en parallèles et en enseignements.

\section{Déjà vu: Lessons to learn from the import of literacy into Francophone discourse}

Francophone writers or translators turning to terminology banks for help in finding a French equivalent of "literacy" might be bewildered by the array of proposed terms and even more puzzled by the fact that equivalents proposed for single terms ("literacy") and compound terms (e.g., "literacy rate") are different, as shown in Table 1.

The banks do provide some notes to help users make more informed choices, for example indicating which terms are "correct" (TERMIUM: littératie, alphabétisme, lettrisme), "preferred" (IATE: littérisme), "privilégiés" (GDT: littératie, littéracie, alphabétisme, lettrisme), "admitted" (IATE: littératie), of "mininum reliability" (IATE: culture informatique), or to "avoid" (TERMIUM: littératie informatique). They point to distinctions in meanings that must be made (IATE: analphabétisme vs. illetrisme) or that can sometimes be made (GDT: analphabétisme vs. illetrisme). TERMIUM and GDT also indicate 


\section{Table 1}

French equivalents of "literacy" proposed by terminology banks

\begin{tabular}{|c|c|c|c|c|}
\hline \multirow[t]{2}{*}{ English } & \multicolumn{4}{|c|}{ French equivalents proposed by: } \\
\hline & TERMIUM & GDT & IATE & FranceTerme \\
\hline literacy & $\begin{array}{l}\text { littératie, } \\
\text { alphabétisme, } \\
\text { littéracie, } \\
\text { lettrisme, } \\
\text { littérisme }\end{array}$ & $\begin{array}{l}\text { littératie, } \\
\text { lettrisme, } \\
\text { lecturisation }\end{array}$ & $\begin{array}{l}\text { littérisme, } \\
\text { aptitude à lire et } \\
\text { à écrire, } \\
\text { littératie }\end{array}$ & $\begin{array}{l}\text { lettrisme } \\
\text { (replacing } \\
\text { littérisme) }\end{array}$ \\
\hline literacy rate & $\begin{array}{l}\text { taux d'alphabé- } \\
\text { tisation }\end{array}$ & $\begin{array}{l}\text { taux } \\
\text { d'alphabétisme }\end{array}$ & $\begin{array}{l}\text { taux d'alphabé- } \\
\text { tisation }\end{array}$ & $\emptyset$ \\
\hline $\begin{array}{l}\text { literacy } \\
\text { campaign }\end{array}$ & $\begin{array}{l}\text { campagne } \\
\text { d'alphabétisa- } \\
\text { tion } \\
\end{array}$ & $\begin{array}{l}\text { campagne } \\
\text { d'alphabétisa- } \\
\text { tion }\end{array}$ & $\begin{array}{l}\text { campagne } \\
\text { d'alphabétisa- } \\
\text { tion }\end{array}$ & $\emptyset$ \\
\hline illiteracy & analphabétisme & $\begin{array}{l}\text { analphabétisme, } \\
\text { illettrisme }\end{array}$ & $\begin{array}{l}\text { analphabétisme } \\
\text { (functional } \\
\text { illiteracy: } \\
\text { illettrisme or } \\
\text { analphabétisme } \\
\text { fonctionnel) }\end{array}$ & $\emptyset$ \\
\hline
\end{tabular}

- TERMIUM (Canada, n.d.): The Government of Canada's terminology and linguistic data bank.

- GDT (Quebec, 2012): Grand dictionnaire terminologique, by the Office québécois de la langue française.

- IATE (European Union, 2019): InterActive Terminology for Europe, European Union's inter-institutional terminology database.

- FranceTerme (France, n.d.): terminology recommended by the Journal officiel de la République française. In FranceTerm, littérisme was the preferred equivalent for "literacy" from 2005 to 2017, when it was replaced by lettrisme.

These terminology banks were consulted in July and August 2018.

when a term is specific to Canada (TERMIUM: alphabétisme) or France (TERMIUM, GDT: lettrisme, littérisme). All banks provide definitions and sources for them.

As shown in Figure 1, a tool such as TradooIT provides further guidance for cross-linguistic term matching by extracting equivalents from terminology banks and a bilingual corpus of translated texts available online (mostly from governmental and international organizations). It also outputs frequency information and referenced, contextualized bilingual concordances taken from the corpus. One learns, for example, that alphabétisation (14,369 occurrences) is much more frequent than littératie $(3,492)$ or alphabétisme $(412)$ in the corpus, 


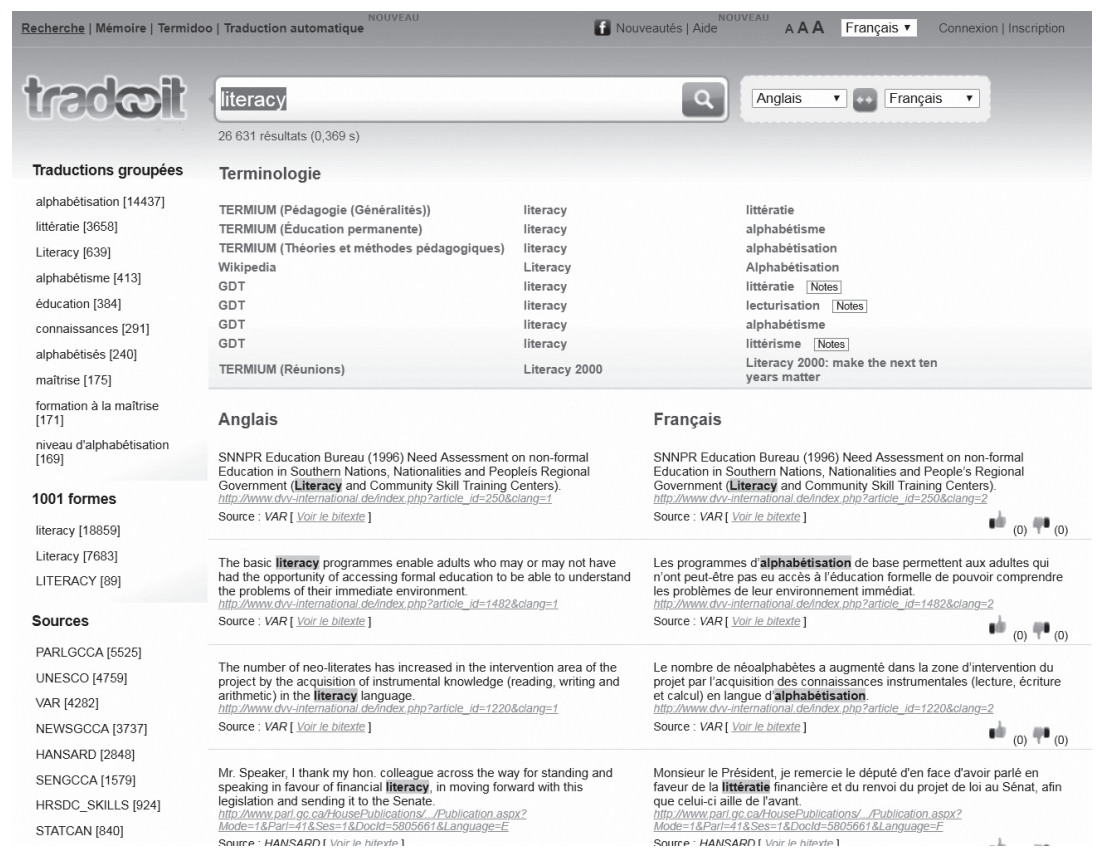

Figure 1

Screenshot showing search results for "literacy" in TradooIT (August 2018)

and one is alerted to less obvious equivalents (e.g., maîtrise de l'information for "information literacy").

These terminology banks, or TradooIT, however, do not do justice to the wealth of vocabulary that has developed in Francophone education discourse to refer to reading and writing practices (pratiques lecturales, scripturales, lecturo-scripturales, pratiques littéraciques, pratiques littéraciées, pratiques de lecture, d'écriture, de littératies), reading and writing knowledge (savoirlire, savoir-écrire, savoir-lire-écrire, compétences en/de lecture, en/d'écriture, habilités en/de lecture, en/d'écriture, capacité en/de lecture, en/d'écriture), and attitudes or "ways of relating" to reading and writing (rapport à l'écrit), among others.

How did we arrive at this terminological maze (not to say mess)? What may have led to the addition of littératie (or littéracie) to an already rich Francophone vocabulary? And how can we help student writers in education appropriate such a vocabulary as they are exposed to readings in English and French (and possibly other languages)?

In advocating for its usefulness in French educational contexts, Delcambre 
(2012) traces the emergence of the notion of "literacies" in France's didactique du français back to the late 1990s-early 2000s and attributes this emergence to two main influences:

- the exposure of French education scholars to (New) Literacy Studies consequent to translations, colloquia, and special issues drawing attention to foundational work by, among others, Jack Goody and Brian Street

- the use of the term in OECD survey reports (most likely translated from English).

The notion, however, had already caught the interest of some Francophone scholars earlier, notably in Canada, where it was explicitly defined as early as 1985 (Hébert \& Lépine, 2013; Painchaud, 1992), but also in France (Chiss, 2004). Another motivation given for the import of "literacy" into French was the need to have a positive counterpart to what illettrisme (1970s) or analphabétisme (early 20th century) refers to negatively (Barré-De Miniac, Brissaud, \& Rispail, 2004, p. 8). It is noteworthy that in English, too, "illiteracy" (middle 17th century, from "illiterate", itself from the Latin literatus) predates "literacy" (late 19th century), suggesting that interest in literacy emerged as a reaction to illiteracy; indeed, "literacy" is thought to have emerged in English in the context of a campaign against illiteracy (Delcambre, 2012, p. 25).

Despite its advocates, the import of "literacy" into French discourse, initially in quotation marks in its English form (Chiss, 1998), then as a literal translation in a French guise, was not without some resistance. Variants in spelling still coexist, with some authors preferring littéracie in recognition of the English filiation (e.g., Delcambre, 2012; Rispail, 2011), while others privileging littératie to signal that the term is not simply transposed but also adapted to Francophone contexts (Glorieux, 2015, p. 40). Jaffré (2004) has pointed out that both -cie and -tie endings are common in French (éclaircie, nécromancie, pharmacie, superficie; aristocratie, amnistie, bureaucratie, démocratie). Littératie, however, seems to be getting the upper hand over littéracie (it is the only spelling given in Le Robert Illustré, 2019; see also Figure 1). Most authors agree that doubling the " $\mathrm{t}$ " is more in keeping with French usage (lettre, littéral, littéraire, littérature,), and litéracie, once popularized by UNESCO (Jaffré, 2004), appears to have fallen out of use.

Spelling aside, one of the main obstacles to the spread of littératiellittéracie into French usage was that it competed with alternatives (alphabétisme, alphabétisation) that had already been popularized by the documentation produced by UNESCO and other organizations in their efforts to address illiteracy (Jaffré, 2004). There was, and still is, a push-back from France's standardization agencies, reflected in FranceTerme's endorsement of littérisme until 2017, and now lettrisme (both of improbable destiny), and echoed in public 
commentaries mocking the anglicism. Despite a more positive reception within academic circles, advocacy for the notion was also met with some skepticism. Reuter (2003) warned against the dressing up of old, questionable ideas in flashy trappings ("oripeaux modernes" p. 12). Ten years later, Hébert \& Lépine (2013, p. 43) concluded their comprehensive review of the use of littératie in Francophone publications from 1985 to 2011 with a similar cautionary observation: that those who advocate the use of litteratie tend to use it in their research in the restrictive, traditional sense of ability to read and write, as a substitute for habilités de lecture-écriture, ostensibly, Hébert and Lépine surmise, because it is the fashionable English buzzword of the time. Conversely, the very strength of the notion - its expressive economy and yet immense meaning potential — is also seen as its main weakness: lack of precision and limited explicatory power (Reuter, 2003; Thévenaz-Christen, 2011).

Overcoming these initial (and remaining) concerns, the notion of littératie now appears to be well-established ("largement circulante," Molinié \& Moore, 2012, p. 4) in Francophone academic discourse, with a consensus emerging that it opens new ways of thinking about reading and writing at home, in school, and in society (Barré-De Miniac et al., 2004). Hébert \& Lépine (2013) have identified ten "added values" from the definitions given by Francophone scholars that champion it. Rather than reviewing them all, I will highlight three types of affordances that I see as particularly fruitful.

First, in terms of theory development, the term affords new perspectives, drawing attention to the relationships between and among reading and writing, oral and other modes of meaning-making, competences and practices. Importantly, it helps to conceive of literacy as emerging from the interrelationship between the competences that individuals develop in particular culturalhistorical contexts, and the social recognition, within those contexts, of these competences qua competences; this dual — individual and social — nature of literacy development is central in understanding the conditions for the transfer, or mobility, of literacy across linguistic and cultural contexts (Gentil, 2011; Painchaud, 1992). Relatedly, the notion of "literacy" helps to conceptualize reading and writing development in its relation to language development in multilingual contexts. Once the notion of littératie is incorporated into the Francophone conceptual horizon, it becomes easier to bring in a derived notion such as bilittératie, from "biliteracy," which Hornberger and Skilton-Sylvester (2003) see as "the conjunction of literacy and bilingualism" (p. xiv). This carves out a space for a new field of inquiry at the intersection of literacy and bilingualism studies, that can be extended to studies in plurilingualism and bi/multilingual education (plurilittéracie, Moore, 2006; pluriliteracies, García, Bartlett, \& Kleifgen, 2007; see also Belhadj Hacen \& Delcambre, 2015).

This is the second type of affordance that littératie offers: the potential 
not only to generate conceptual shifts but also to bring or to "label" new fields of inquiry into being. Delcambre (2012), Glorieux (2015), and Pollet (2014) retraced how the concept of littératie(s) universitaire(s), seen as a type of littératie, enabled the emergence of a new field in France and Belgium with links to Britain-based Academic Literacies and US-based Composition Studies. Such conceptual and disciplinary reconfigurations in turn open up a third type of affordance: to generate changes in social practice, particularly educational practice. Pollet offers a promising example of how littératies universitaires, as a concept and an orientation, can inform a curriculum that aims to initiate university students into reading and writing in their disciplines (on the curricular impact of a littératie perspective in France or in Québec, see also Chiss, 2004; Hébert \& Lepine, 2013).

The profuse terminology around literacy in French thus reflects the push and pull that surrounded the emergence of a new vocabulary, around littératie, amidst a well-established and officially sanctioned vocabulary (alphabétisation, illettrisme). The introduction of littératie, with all its orthographic variants, within academic circles, resulted in a conspicuous mismatch, in France especially, between academic and everyday discourses about reading and writing, confining littératie to an ivory tower for a good decade. This may be changing, however, as the new term seems to be making inroads into public discourse, for example when journalists for Le Monde interview literacy experts or relate findings of OECD reports. Indeed, while Jaffré (2004, p. 25) noted its absence from French language dictionaries, littératie now appears in the 2019 edition of Le Robert illustré (it remains absent from Le Larousse online).

The insight gained from the complex reshuffling of French vocabulary related to "literacy" under the influence of English is worth bearing in mind when considering what to do with a much more recent coinage: "translanguaging." It is quite clear that importing terminology from another language goes beyond mere questions of borrowings and linguistic equivalences. At play are debates about benefits and drawbacks - for whom? - and underlying these, the conditions of the circulation of ideas across linguistic traditions (cf. Liddicoat \& Zarate, 2009), and the ripple effects that such circulation can have on discourses, praxis, and people. This short history of the assimilation of "literacy" into French discourse also illustrates the limitations of terminology banks for plurilingual writers and translators: while they can serve useful referencing and normalizing functions, they provided only a partial and delayed glimpse into changing discourses. 


\section{Translanguaging in translation-Trans ... quoi?}

Cette courte rétrospective de l'introduction de «littératie » souligne l'intérêt, et aussi les limites, des ressources qu'offrent la traductologie et la terminologie multilingue aux scripteurs universitaires plurilingues, tout en attirant l'attention sur les conditions et les enjeux de la réception d'un concept dans un nouvel espace linguistique. Fort de ce parcours, le temps est maintenant venu de se pencher sur la question de savoir comment traiter translanguaging en français. Je procéderai en trois étapes. D'abord, il importe de rechercher les emplois et traductions existantes, dans les portails de publications universitaires et en tirant profit des outils de l'ingénierie linguistique mentionnés précédemment (banques terminologiques, concordanciers bilingues, traducteurs en ligne). Ensuite, je tenterai de m'appuyer sur les méthodes de la terminologie pour cerner les contours de la notion de translanguaging, en anglais, dans le domaine de l'éducation, dans une double démarche onomasiologique (du concept à sa réalisation linguistique) et sémasiologique (de l'appellation au concept qu'elle recouvre) (L'Homme, 2008). Le point de vue sémasiologique nous incitera à repérer les formes, les fonctions et les dérivations du terme, puis à le décomposer en morphèmes constitutifs. La perspective onomasiologique nous invitera à situer translanguaging dans un champ notionnel, en le mettant en lien avec d'autres notions qui ne sont pas nécessairement proches dans la forme (par exemple, « compétence plurilingue et pluriculturelle, » «médiation linguistique, » «mise en langage, » «méso-alternance»). C'est cette démarche onomasiologique qui, finalement, nous conduira à envisager différentes formes équivalentes, en français, des acceptions du terme translanguaging relevées en anglais.

\section{Traductions et emplois existant en français}

À l'heure de la rédaction de ce texte (août 2018), Translanguaging ne figure pas dans les banques terminologiques canadiennes (GDT, TERMIUM) ou européennes (FranceTerme, IATE). Il n'apparaît pas non plus dans TradooIT. Linguee, un concordancier bilingue qui, comme TradooIT, extrait des équivalents à partir de sites bilingues, ne donne que trois résultats (cf. Figure 2).

Les deux premières concordances proviennent de la même source, un rapport bilingue archivé sur le site du Commissariat aux langues officielles (Rehorick, 2011), vraisemblablement composé en anglais puis traduit. Translanguaging y est rendu par «trans-apprentissage linguistique », qui laisse perplexe. «Apprentissage translinguistique» aurait été plus parlant et plus proche de translanguaging (du moins dans l'une des acceptions du terme, cf. infra). La troisième concordance provient du site du Congrès 2011 des sciences humaines, mais la page d'où elle est tirée n'est plus accessible; elle met en corres- 


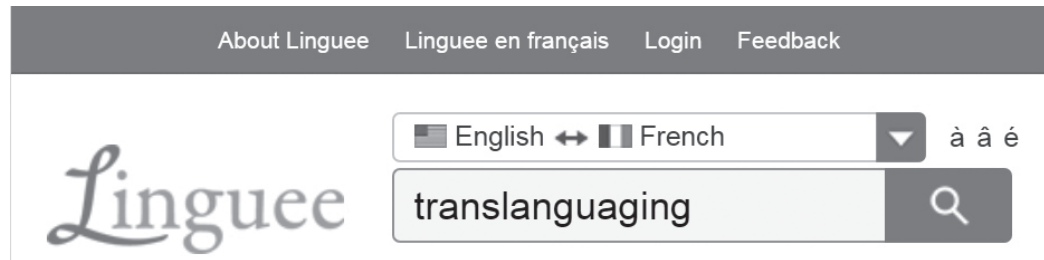

vexternal sources (not reviewed)

In 'translanguaging', the input (reading or
listening) tends to be in one language, and
the output (speaking or writing) in the other
language.
$\square$ ocol.gc.ca
$\triangle$ Translanguaging" is one teaching strategy
used in this setting, and is described as
"...the hearing or reading of a lesson, [...]
$\square \rightarrow$ ocol.gc.ca

$\triangle[\ldots]$ Enemy, a ground-breaking account of the transnational and translanguage character of literature during the Hundred Years War.
En trans apprentissage linguistique, l'intrant (lire ou écouter) tend à être dans une langue, tandis que l'extrant (parler [...]

$\triangle$ Le « trans apprentissage linguistique » est l'une des stratégies d'enseignement utilisées dans ce contexte et se décrit [...]

$\square \rightarrow o c o l . g c . c a$

$\triangle[$ [...] Enemy, un compte-rendu innovateur du caractère transnational et interlingual de la littérature pendant la guerre de Cent Ans.

$\leftrightarrow$ congress2011.ca

\section{FIGURE 2}

Saisie d'écran montrant les résultats d'une recherche pour translanguaging dans

Linguee (août 2018)

pondance translanguage avec interlingual, qui serait sans doute mieux rendu par « interlingue » (cf. « bilingue, » « plurilingue »).

Des recherches du terme translanguaging dans les portails de sciences humaines et sociales de langue française (Cairn, Érudit, Persée), ou dans les publications en français des portails anglophones (ERIC, LLBA), ne donnent que peu de résultats : au plus neuf dans Cairn et neuf dans érudit, et le plus souvent le terme n'apparaît que dans le titre d'une référence citée (date de consultation : août 2018). Parfois, la citation fait l'objet d'un commentaire dans le texte. Dans ce cas, le mot est soit laissé tel quel en anglais, soit reformulé en français, ce qui permet de relever les expressions suivantes : «stratégies dites translangagières » (Rughoonundun-Chellapermal, 2017), « pédagogie translinguistique » (Blanchet, Clerc et Rispail, 2014, qu'ils mettent en parallèle à la « pédagogie interlinguistique »), « pratiques translangagières » (Trépos et al., 2016), « pratiques translinguistiques » (Maynard et Armand, 2016, terme qui serait «adapté de celui de compétences translinguistiques utilisé par Candelier 
et De Pietro, 2008 », p. 138). Enfin, on retrouve «trans-apprentissage linguistique » dans le résumé français d'une thèse en anglais de l'Université McGill (Chukly-Bonato, 2016). On observe donc une concurrence entre « translinguistique » et «translangagier, » sur laquelle je reviendrai.

Autre piste : les traducteurs en ligne. Google Translate propose translanguaging comme équivalent français - les algorithmes seraient-ils pris à défaut, ou s'agirait-il de la traduction la plus fréquente ? DeepL offre deux propositions plus intéressantes : «translangagerie » et « translinguisme» (en plus de «traduction» et «traductibilité »). Dernière source : les publications du Conseil de l'Europe, qui ont l'intérêt d'être disponibles in extenso en anglais et en français. Translanguaging n'apparaît pas dans le Cadre européen commun de référence (CECR) pour les langues (Conseil de l'Europe, 2001), mais il est mentionné brièvement, pour ne pas dire lapidairement, dans le volume complémentaire paru récemment (Conseil de l'Europe, 2018) :

Par une curieuse coïncidence, 1996 est aussi l'année où le terme de 'translanguaging' est apparu (en rapport avec l'enseignement bilingue au Pays de Galles). Le 'translanguaging' est une action entreprise par des personnes plurilingues et impliquant plus d'une langue. Une foule d'expressions identiques existent à présent, mais elles sont toutes incluses dans le terme plurilinguisme. (p. 28)

Cet extrait souligne bien la préférence du Conseil de l'Europe pour une terminologie centrée sur le «plurilinguisme» et la «compétence plurilingue et pluriculturelle »; translanguaging, non traduit, n'est pas retenu. Le terme est cependant mentionné dans une autre publication liée au CEFR, mais une fois seulement, dans un tableau sur les notions de plurilinguisme sous-jacentes au CEFR ; il est alors traduit par «translangage» (North et Piccardo, 2016, p. 31).

Finalement, un.e évaluateur.trice anonyme m'a signalé des travaux que je n'avais pas trouvé dans les portails francophones :

- ROMtels, un projet ERASMUS ${ }^{4}$ visant à améliorer l'éducation d'enfants de gens du voyage et dans le cadre duquel des guides ont été formulés en quatre langues, dont le français, par l'équipe de Nathalie Auger, pour expliquer la «pédagogie translangagière » au personnel éducatif

- les travaux de Joëlle Aden $(2012,2013)$ sur le translangager et de Sandrine Eschenauer (2014) sur la translangageance.

\footnotetext{
${ }^{4}$ Students in Erasmus programs travel and study in the different countries of the European Community and very often find themselves studying in English in the countries where they stay. These exchanges are named in honour of Erasmus of Rotterdam, who advocated travel and stays in different parts of Europe.
} 


\section{Formes, fonctions, et dérivés}

Avant de commenter les traductions existantes, ou le choix de ne pas traduire, puis de considérer d'autres traductions possibles, il est important d'observer que translanguaging, en anglais, s'emploie d'une façon très souple, comme en témoigne le Tableau 2. Comme nom, il apparaît aussi bien dans des syntagmes sujets que compléments, parfois avec un déterminant («their translanguaging »). Il est très productif aussi comme nom à valeur adjectivale («translanguaging practices »). Il est également utilisé comme un verbe («to freely translanguage»), ce qui s'accorde bien avec l'une des dimensions centrales de la notion, à savoir que le langage est avant tout un processus, une activité, plus qu'un système ou un code. Cependant, cet emploi verbal reste assez occasionnel, et en concurrence avec une forme nominalisante : « use translanguaging » (Gentil, 2017). Enfin, García et Lin (2017) utilisent une forme dérivée : «translanguaged» (p. 125). Pour rendre opératoire une telle notion en français, à supposer que cela soit souhaitable, il me semble donc important de trouver des équivalents nominaux, verbaux et adjectivaux (à l'instar de « littératie, » dont on dérive parfois un adjectif : « littéracique » ou « littératié »).

TABLEAU 2

Emplois de translanguaging en anglais

\begin{tabular}{ll}
\hline \hline Classe & Exemples \\
\hline Nom & Translanguaging is ... \\
& $\ldots$ their translanguaging ... \\
& $\ldots$ work/research on translanguaging $\ldots$ \\
& $\ldots$ how [they] use translanguaging .... \\
\hline
\end{tabular}

Nom à fonction $\mathrm{a} \sim$ approach/perspective/theory/lens, theoretical framework adjectivale

$\sim$ space, $\sim$ practice $(\mathrm{s}), \sim$ pedagogical strategies, $\sim$ instructional materials

Verbe "university students ... are encouraged to freely translanguage in the Sepedi language, local language varieties, and South African English in order to ..." (Schwinge, 2017, p. 155)

Adjectif dérivé "the translanguaged multimodal assessment" (García et Lin, 2017, p. 125)

Note : Ces emplois ont été relevés dans les huit volumes de l'Enclyclopedia of Language and Education (Cenoz et al., 2017). 


\section{Décompositions de la notion et notions dérivées}

Remarquons finalement que translanguaging se décompose en trans-, languag-, et -ing. Wei et García (2017, p. 233) attirent l'attention sur l'importance du préfixe trans-, en lien selon eux avec les valeurs transgressives, transformatives et transdisciplinaires de l'orientation, ainsi que les idées de trans-system, trans-space. Le préfixe, qui semble avoir le vent en poupe dans la littérature éducative anglophone, renvoie à plusieurs notions circulantes : transnational, translation et translingual (lequel évoque tout un courant pédagogique dans l'enseignement universitaire de la rédaction aux états-Unis : Atkinson et al., 2015). Trans- ne cause pas de problème de traduction majeur, puisque, dérivé du latin, il existe en français avec des valeurs voisines d'au-delà, d'à travers, de passage ou de changement. Languag- renvoie bien sûr au champ notionnel de la langue et du langage (dont les notions de bi/pluri/multilinguisme), mais sans distinction explicite entre langue(s) et langage(s). Tout aussi important, le suffixe -ing, très productif en anglais et suggérant très bien l'idée de processus (doing, acting, believing, becoming, etc.), est essentiel à la notion de translanguaging, tout en la mettant en rapport avec celle, voisine, de languaging (Swain, 2006). Il soulève quelques difficultés de traduction, notamment parce qu'il se substitue facilement à d'autres terminaisons pour dériver verbes et adjectifs (they translanguage, translanguaged assessment).

\section{Traductions possibles selon les acceptions de translanguaging}

Écueil supplémentaire : translanguaging est une notion non seulement polysémique, mais aussi très évolutive. N'en déplaise aux terminologues, cette réfractivité à tout embrigadement définitoire est même revendiquée par les partisans : «the essential assumption is that there can be no exact or essentialist definition as the meaning of translanguaging will become more refined and increasingly clarified, conceptually and through further research » (Lewis et al., 2012, p. 642).

Heureusement, la généalogie de la notion proposée par Lewis et al. (2012), complétée par les rétrospectives plus récentes de García (García et Lin, 2017; Wei et García, 2017), permettent de mieux cerner les acceptions du terme, dans le domaine de l'éducation, depuis son introduction en gallois puis en anglais par Williams (1994, cité dans Lewis et al., 2012, p. 643) et Baker (2001, 2003). Ces acceptions sont résumées dans le tableau 3 , dans l'ordre chronologique où elles sont apparues, avec pour chacune, des traductions envisageables. Pour repérer ces acceptions, il s'est avéré utile de porter une attention particulière à la catégorie, soulignée, dans laquelle chaque définition inclut le terme, ce que les lexicographes appellent «l'incluant» et ce qui constitue l'ancrage de la définition (Dubuc, 2002, p. 100). Dans son acception originelle (la définition [1a] 


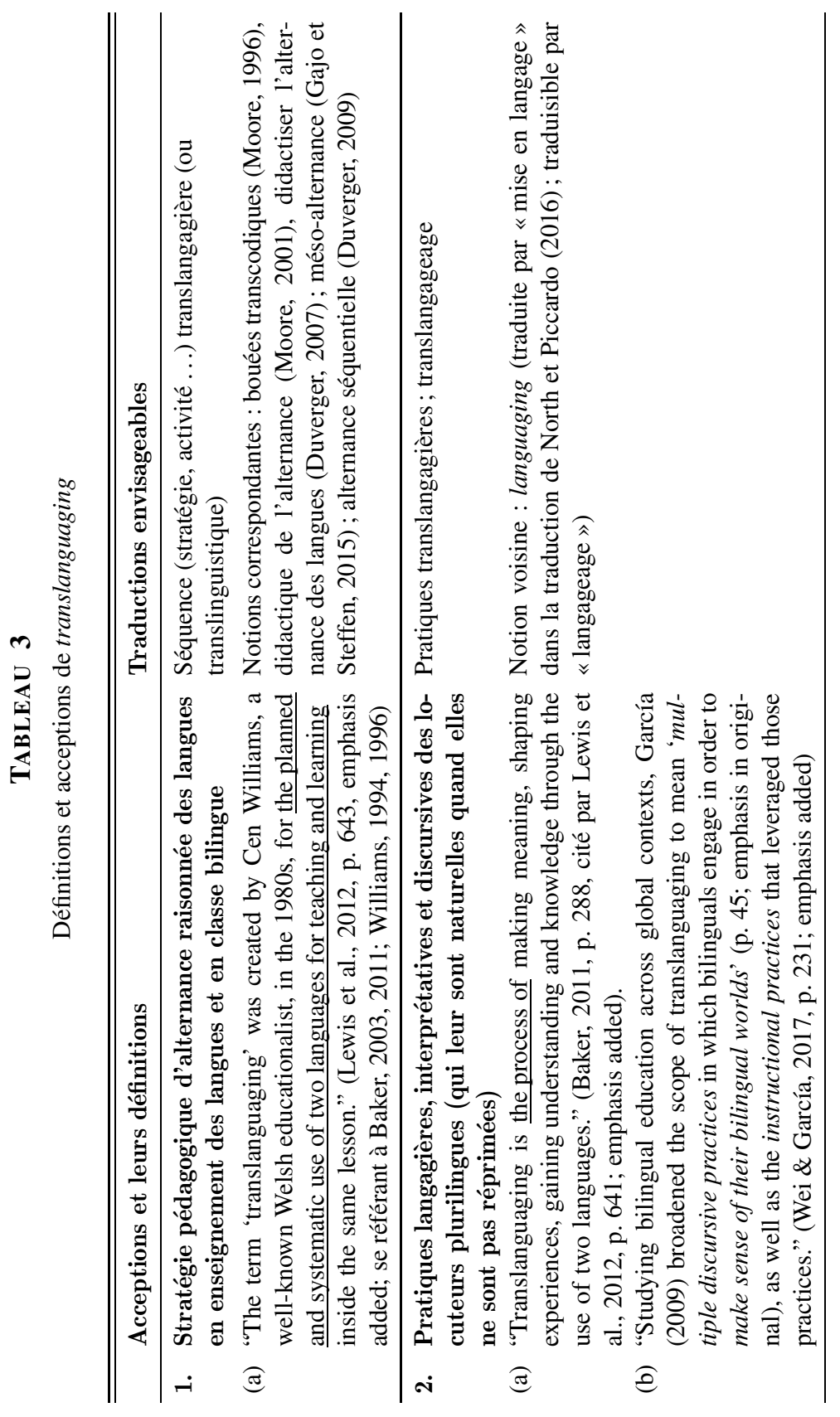




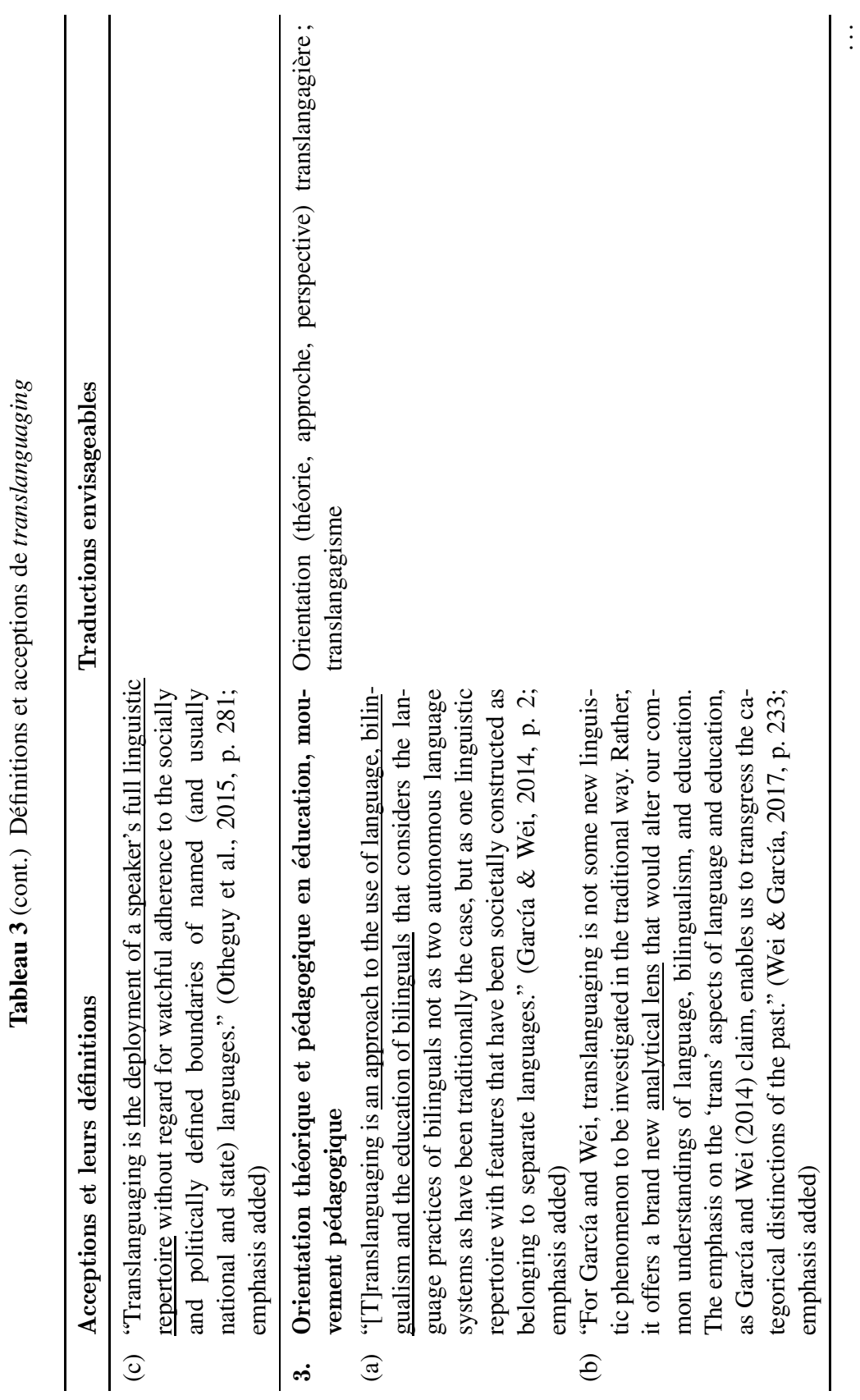




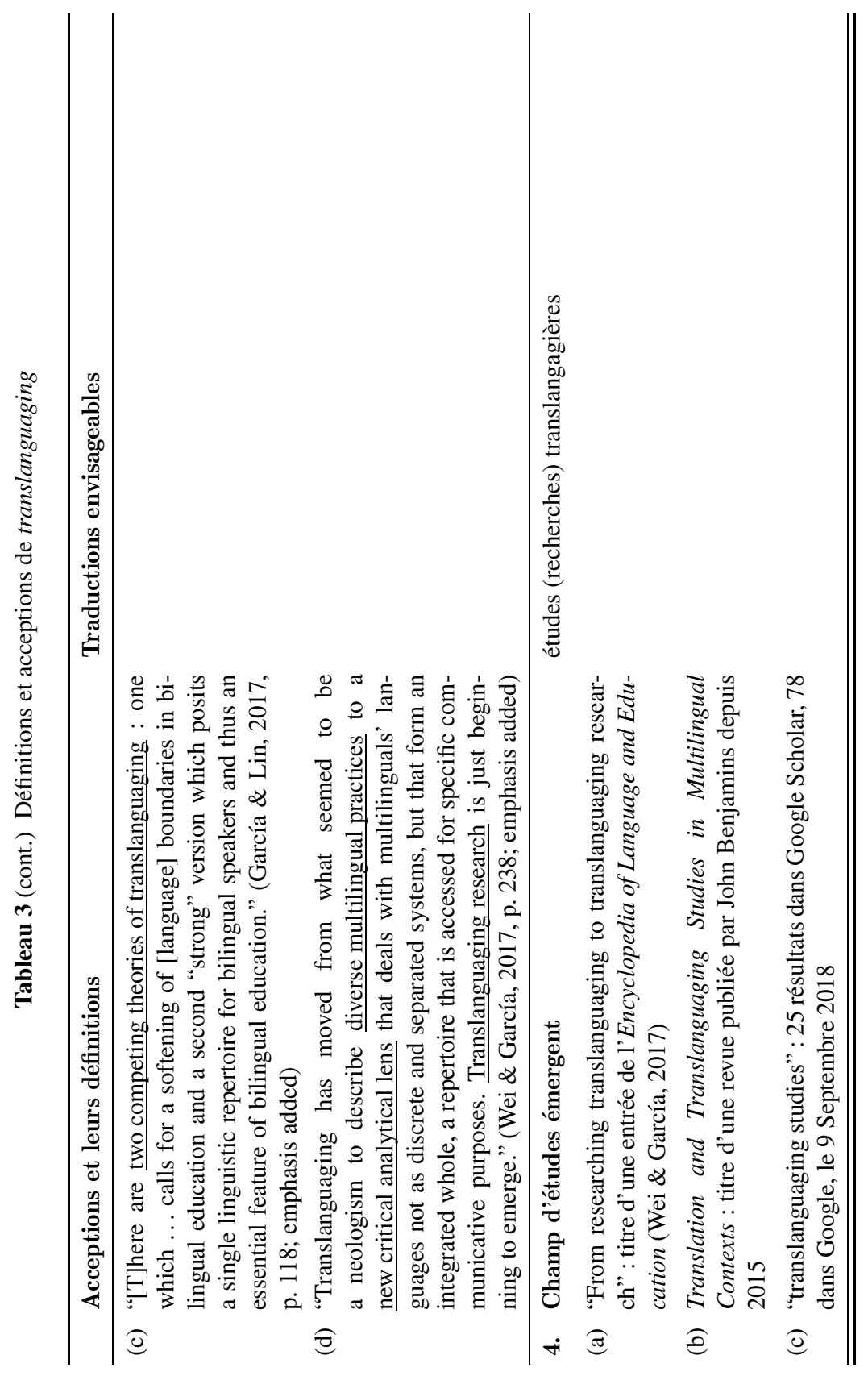


du tableau 3), le translanguaging réfère ainsi à une stratégie pédagogique en classe bilingue d'un type particulier, utilisée au pays de Galles, consistant à planifier une alternance des langues au cours d'une séquence d'enseignement, en donnant de l'information à lire ou à écouter sur un sujet dans une langue pour ensuite la reprendre en production orale et écrite dans une autre langue. Cette stratégie est l'inverse de celle préconisée dans les programmes canadiens d'immersion, qui repose sur une séparation stricte des langues, par matières, par plages horaires et par espaces monolingues dédiés, du moins en théorie (Baker, 2001). On pourra traduire cette première acception par « séquence (ou stratégie) translangagière, » tout en remarquant que les didacticiens francophones ont développé, parallèlement, tout un vocabulaire pour décrire ce type d'alternance dont l'intérêt pédagogique ne leur a pas échappé. Gajo et Steffen (2015) font d'ailleurs un rapprochement explicite entre la «méso-alternance » et « la notion de translanguaging» (p. 476). «Bouées transcodiques, » «alternance séquentielle, » «didactique de l'alternance, » et «didactiser l'alternance » ont également été relevés (par exemple, Duverger, 2007, 2009; Moore, 1996, 2001). Dans cette acception d'alternance délibérée de langues conçues comme des systèmes linguistiques autonomes, «translinguistique » pourrait éventuellement se substituer à «translangagier»; j’y reviendrai.

L'évolution vers la seconde série d'acceptions est attribuable à Ofelia García (2009), qui voit dans translanguaging le mode opératoire des locuteurs plurilingues, lesquels seraient naturellement portés à utiliser l'ensemble de leur répertoire linguistique dans leur appréhension du monde s'ils n'étaient pas contraints à en inhiber une grande partie en contexte monolingue mononormatif. Cet incluant commun de «processus » ou de «pratiques » (process, practices, deployment) propres aux locuteurs plurilingues permet de regrouper les définitions (2a), (2b) et (2c), malgré quelques nuances, sous la bannière des « pratiques translanguagières ». La définition (2b) comprend en fait deux incluants, «multiple discursive practices » et «instructional practices », ce qui permet de faire le lien entre les acceptions (1) et (3). Selon cette définition, la notion de translanguaging recoupe non seulement les pratiques translangagières spontanées des plurilingues mais aussi la façon dont elles devraient être mises à profit («leveraged») en classe. Dans cette acception élargie, la notion de translanguaging conserve donc sa dimension pédagogique initiale, mais les stratégies pédagogiques proposées par García (2009; voir aussi Wei et García, 2014) ne sauraient se limiter au modèle gallois de l'alternance.

C'est ainsi que la notion évolue vers un troisième sens, celui d'une orientation théorique et pédagogique, voire d'un mouvement pédagogique, regroupant un éventail large et ouvert de stratégies pédagogiques du moment qu'elles restent compatibles avec l'orientation générale (définitions [3a] à [3d]). Il importe donc de distinguer la pédagogie translangagière galloise, soit une façon 
particulière de didactiser l'alternance, de l'orientation translangagière, plus globale (et globalisante), qui prend forme dans le prolongement des travaux de García et de Wei. Cette orientation comprendrait, selon García et Lin (2017, voir définition [2c]), deux versions concurrentes : une version faible, qui enjoindrait à assouplir les frontières linguistiques érigées entre les langues dans l'enseignement des langues et en classe bilingue, et une version forte, qui conteste l'existence même de ces frontières linguistiques dans les représentations mentales sous-jacentes aux répertoires langagiers des locuteurs plurilingues. Remarquons à ce sujet que la principale différence entre la définition (2a), attribuée à Baker (2011), comparée aux définitions (2b) et (2c), de García, est qu'elle limite translanguaging à l'emploi de deux langues, ce qui présuppose un dénombrement, et donc un découpage du répertoire linguistique plurilingue en des langues distinctes; or un tel présupposé est incompatible avec la version forte de l'orientation translangagière préconisée par García.

On pourrait baptiser cette «perspective translangagière» sous le vocable de «translangagisme» si le suffixe -isme n'était pas empreint de connotations péjoratives (qui pourraient plaire aux détracteurs). Quoi qu'il en soit, étant donné l'ampleur que prend cette orientation dans les recherches en éducation plurilingue, aux états-Unis notamment, dont témoigne un foisonnement de publications, on peut se demander si on n'assisterait pas à l'émergence d'un nouveau champ d'études, voire à une reconfiguration des recherches en éducation bilingue, sous le label «études translangagières ». Ce processus n'est peutêtre pas aussi avancé que pour d'autres nouveaux champs d'études (comme literacy studies, « littératies universitaires, » «études genre » mentionnées supra), mais plusieurs indices témoignent de cette évolution : le titre d'une entrée encyclopédique, «From researching translanguaging to translanguaging research » (Wei et García, 2017); l'apparition d'une nouvelle revue, Translation and Translanguaging in Multilingual Contexts, publiée par John Benjamins (2015), dans le but d'offrir un forum à un « burgeoning field of scholarly enquiry and practice»; et l'émergence de translanguaging studies sur la toile (une vingtaine de résultats relevés dans Google Scholar en septembre 2018, environ 80 dans Google).

Comme on le voit, l'adjectif «translangagier» s'accommode très bien de toutes ces acceptions. La structure nom + «translangagier $»(+$ adjectif) présente aussi l'intérêt non négligeable de préciser l'acception voulue, ce qui permet de démêler les multiples sens de translanguaging : pédagogie translangagière galloise, pratiques translangagières, orientation translangagière, études translangagières. Autre argument en faveur de ce néologisme : il est apparu spontanément sous la plume des quelques auteur.e.s francophones y faisant référence. Il me semble préférable à «translinguistique» pour deux raisons principales. En premier lieu, rimant avec «linguistique, » « translinguistique » 
évoque une conception plus traditionnelle des langues comme systèmes, en décalage avec les orientations sous-jacentes à la notion de translanguaging, excepté peut-être dans son acception originelle de pédagogie d'alternance codique (acception 1 dans le tableau 3 ). C'est pourquoi «translinguistique » serait envisageable comme équivalent de translanguaging essentiellement dans cette acception. Dans les autres acceptions, «translangagier» me semble conceptuellement plus proche de translanguaging, et donc mieux motivé et plus transparent. Quoiqu'une distinction entre «translinguistique » et «translangagier» pourrait marquer ce glissement de sens et d'orientation, l'utilisation d'un seul terme me semble préférable autant par souci de parcimonie que pour essayer de faire correspondre, en français, la polysémie de translanguaging en anglais (tout en prenant soin de préciser le sens visé).

En deuxième lieu, l'intérêt de «translangagier » réside en son fort potentiel dérivatif : «translangager, » «translangageage, » « translangagisme, » «translangageries. » Certes, ces dérivés demanderaient à l'oreille de s'y accoutumer, ce dont l'usage jugera, mais «translangager» et «translangageage, » en particulier, méritent d'être envisagés car ils rendent très bien une dimension clé de translanguaging, à savoir que si la langue, ou le langage, devrait être considéré avant tout comme une activité humaine, un processus interprétatif, une pratique sociale, il devrait s'utiliser comme un verbe (to language, to translanguage) ou un prédicat nominal (languaging, translanguaging) plus que comme un nom. C'est en cela que la notion de translanguaging peut être rapprochée de celle de languaging, pourtant élaborée indépendamment (Swain, 2006). Langager, ou le langageage, c'est utiliser les ressources du langage non seulement pour mieux comprendre, clarifier, élaborer sa pensée, mais aussi pour construire du sens et des savoirs, en formulant à voix haute ou à l'écrit; translangager, ou le translangageage, c'est faire la même chose, mais en contexte plurilingue et translingue, en puisant dans les ressources de plusieurs traditions linguistiques, en jonglant avec les langues et en combinant leurs potentiels de signification. Traduire languaging par « mise en langage » (North et Picardo, 2016) rend assez bien l'idée du concept mais cette traduction me semble avoir deux défauts. D'abord, l'expression «mise en langage » pourrait suggérer que la langue ne ferait que mettre en mots des idées préétablies; or langager, c'est non seulement exprimer du sens mais aussi le créer par et dans l'acte de parole. Ensuite, traduire languaging par «mise en langage » gomme le lien qu'il entretient avec translanguaging; la dérivation verbale ou en -age a l'avantage de mettre les deux concepts en rapport tout en soulignant l'idée d'action, l'une des valeurs du suffixe (cf. « affinage, » « atterrissage, » «babillage, » « baragouinage, » « chuchotage, » « magasinage, » etc.).

On pourrait bien sûr aussi conserver translanguaging en français dans le texte, comme le font plusieurs auteurs, et comme l'ont fait les organisateurs du 
colloque de CCERBAL. Le suffixe en -ing est productif en français, en France notamment, donnant lieu parfois à des usages très éloignés de l'anglais, voire difficiles à concevoir pour des anglophones (cf. brushing, footing, jogging). L'inconvénient d'importer translanguaging tel quel est que, sous cette forme, il est bien moins maniable en français qu'en anglais, puisqu'on ne peut en dériver verbes ou adjectifs; il est plus opaque aussi et donc, en final, moins opératoire, que «translangagier » et ses dérivés (pour des critères de viabilité des néologismes, cf. Dubuc, 2002, p. 130-131).

Cette section ne serait complète sans commenter brièvement le néologisme original de «translangageance» que propose Eschenauer (2014), à partir des travaux d'Aden $(2012,2013)$, son ancienne directrice de thèse, sur le «translangager, » dans le cadre de recherches sur la pratique théâtrale et les activités de médiation (corporelle, linguistique, culturelle) dans une pédagogie des langues visant à développer des compétences à la fois langagières (plurilinguisme, flexibilité linguistique) et émotionnelles (empathie, décentrage, ouverture aux autres). Dans cette optique, les activités translangagières sont conçues comme impliquant non seulement le verbal et les langues, mais aussi le corps et le non-verbal, c'est-à-dire d'autres langages ou systèmes d'expression et de constitution du monde, dont le langage théâtral. De plus, formulée dans un paradigme énactif et émergentiste (Aden, 2017), la didactique du translangager ou de la translangageance que proposent Eschanauer et Aden insiste sur le rôle central de ce mouvement de soi vers l'autre et le monde, d'un dépassement d'un ici vers un au-delà, rendu par le préfixe trans-, dans la constitution d'un sens et d'un espace partagés; translangageance rime donc avec émergence, transcendance, reliance et résonance. Il est intéressant de constater comment, en s'appropriant la notion de translanguaging tout en la traduisant, Eschanauer et Aden l'enrichissent de nouvelles inflexions. Cela dit, je me demande s'il ne serait pas préférable de réserver «translangageance» au concept défini par Eschanauer (2014).

\section{Approche translangagière, translangager, translangageage- What's the added value en français?}

I hope to have shown that, contrary to doubts that francophones themselves can express in this regard, the French language has the expressive resources required to render English notions such as literacy and translanguaging. Strong cases have been made for the added value of introducing littératie into Francophone academic discourse, and the coinage seems to be gaining ground, slowly, in more general usage as well. What could be the added value of translangagier, translangager, and translangageage for Francophone scholars, educators, and students?

One cannot but notice the striking similarities between the translanguag- 
ing pedagogical model developed in Wales and the translanguaging practices of plurilingual writers who must compose in one language from (written and oral) sources in another. As a Francophone researcher interested in pluriliteracy and transliteracy development and a bilingual writer myself, I felt a need for a vocabulary both to describe such literacy practices and to conceive of pedagogies that would address the unique demands that they place on plurilingual writers. "Translanguaging" (along with "biliteracy," "pluriliteracy," "transliteracy") has helped to meet this need in English, and translangagier (with its derivatives and bilittératie, plurilittératies, translittératie) is fulfilling this need in French.

The fast-growing body of English literature about translanguaging has made the notion quite complex and harder to pin down. At the same time, attending to its various meanings offers food for thought. In particular, it helps us think about the demands of translanguaging practice, not only as a challenge for plurilingual writers, but also an opportunity for gaining understanding and knowledge through a plurilingual lens. Without necessarily subscribing to the strong version of translanguaging theory, which contests the psychological reality of bounded language systems in plurilingual minds, thereby questioning the very existence of multilingualism or the very idea of translating across languages (MacSwan, 2017), a translanguaging perspective on plurilingual education and pluri/transliteracy development has much to offer. It calls for rethinking language allocation strategies in bilingual education program, questioning the ban on L1 use in L2 instruction, and reconfiguring disciplinary and curricular arrangements, in ways that best support the academic and language development of plurilingual students. With regard to plurilingual writers in university settings, I have tried to suggest how their translanguaging needs might be best supported by drawing on professional translation training as a means of decompartmentalizing, or complementing, L1 and L2 writing instruction.

It is too early to tell whether the notion of "translanguaging" might give rise to a new field of study, in the same way as "literacy" gave rise to the field of littératies universitaires (Glorieux, 2015). Perhaps, a translanguaging orientation need not develop into a field of study to mediate conversations among the various subfields which might contribute to pluri/transliteracy in university contexts: L1 and L2 writing studies, translation studies, language teaching, and multilingual education in the Anglophone academic landscape; rédactologie, traductologie, didactique de l'écrit, didactique des langues, littératies universitaires, pédagogie universitaire, and enseignement bi/plurilingue in the Francophone world. As the introduction of littératie into Francophone discourse illustrates, questions about what to do with translanguaging in French raise broader questions about the conditions and consequences of the circulation of ideas across national and linguistic contexts. The potential added value that 
"translanguaging" could bring to Francophone scholarship and educational practice should be gauged in light of the existing vocabularies and discourses it could contribute to and possibly displace. The idea that plurilinguals should be encouraged to use and develop their linguistic repertoire at school was already at the core of the didactique plurinormaliste developed in France in the 1980s (Blanchet, 2015). And the critique of the monolingual paradigm that underpins translanguaging scholarship finds a parallel in Francophone scholars' attempt to develop a didactique $d u$ plurilinguisme et du pluriculturalisme from a nonmonolingual perspective in the 1990s (Castelloti \& Moore, 2015; Moore \& Gajo, 2009). Questions about the translation and reception of "translanguaging" into French arise as Francophone scholars are exposed to, and become interested in, Anglophone scholarship. It is striking how translanguaging scholarship in English hardly refers to any work in languages other than English (with the notable exception of Williams' seminal 1994 work in Welsh). This seems at odds with a translanguaging orientation that embraces multiple linguistic repertoires. How could English-medium translanguaging scholarship engage with scholarship in other languages, and what challenges and benefits would be associated with this? In other words, what would translanguaging scholarship look like if it not only talked about translanguaging but also translanguaged itself?

\section{References}

Aden, J. (2012). La médiation linguistique au fondement du sens partagé: vers un paradigme de l'enaction en didactique des langues. Ela. Études de linguistique appliquée, 167, 267-284.

Aden, J. (2013). Apprendre les langues par corps. Dans S. Abdlekader et O. Fertat (dir.), Pour un Théâtre-Monde: plurilinguisme, interculturalité, transmission (p. 109123). Pessac, France: Presses universitaires de Bordeaux.

Aden, J. (2017). Langues et langage dans un paradigme enactif. Recherches en didactique des langues et des cultures. Recherches en didactique des langues et des cultures: les Cahiers de l'Acedle, 14. doi.org/10.4000/rdlc.1085

Ammon, U. (Ed.). (1996). The dominance of English as a language of science: Effects on other languages and language communities. Berlin: Mouton de Gruyter.

Atkinson, D., Crusan, D., Matsuda, P.K., Ortmeier-Hooper, C., Ruecker, T., Simpson, S., \& Tardy, C. (2015). Clarifying the relationship between L2 writing and translingual writing: An open letter to writing studies editors and organization leaders. College English, 77, 383-383.

Baker, C. (2001). Foundations of bilingual education and bilingualism. Clevedon, UK: Multilingual Matters. 
Baker, C. (2003). Biliteracy and transliteracy in Wales: International and critical perspectives. In N.H. Hornberger (Ed.), Continua of biliteracy: An ecological framework for educational policy, research, and practice in multilingual settings (pp. 7190). Clevedon, UK: Multilingual Matters.

Baker, C. (2011). Foundations of bilingual education and bilingualism (5th ed.). Clevedon, UK: Multilingual Matters.

Barré-De Miniac, C., Brissaud, C. et Rispail, M. (dir.). (2004). La littéracie: conceptions théoriques et pratiques d'enseignement de la lecture-écriture. Paris: L'Harmmattan.

Belhadj Hacen, A. et Delcambre, I. (dir.). (2015). Littéraices et plurilinguismes: quelles pratiques? Quels liens? Paris: L'Harmattan.

Blanchet, P. (2015). Plurilinguismes et lit(t)éracies: problématiser les enjeux interculturels, éducatifs et sociétaux. Dans A. Belhadj Hacen et I. Delcambre (dir.), Littéracies et plurilinguismes: quelles pratiques? quels liens? (p. 21-33). Paris: L'Harmattan.

Blanchet, P., Clerc, S. et Rispail, M. (2014). Réduire l'insécurité linguistique des élèves par une transposition didactique de la pluralité sociolinguistique. Pour de nouvelles perspectives sociodidactiques avec l'exemple du Maghreb. Ela. Études de linguistique appliquée, 175, 283-302.

Bourdieu, P. (1994). Raisons pratiques: sur la théore de l'action. Paris: Seuil.

Canada. (n.d.). TERMIUM Plus. www.btb.termiumplus.gc.ca/tpv2alpha/alpha-eng.html?lang=eng

Castellotti, V. et Moore, D. (2015). La compétence plurilingue et pluriculturelle: genèses et évolutions. Dans $\mathrm{Ph}$. Blanchet et $\mathrm{P}$. Chardenet (dir.), Guide pour la recherche en didactique des langues et cultures: approches contextualisées (p. 291302). Paris: éditions des archives contemporaines.

Cenoz, J., \& Gorter, D. (2017). Translanguaging as a pedagogical tool in multilingual education. In J. Cenoz, D. Gorter, \& S. May (Eds.), Encyclopedia of language and education: Language awareness and multilingualism (pp. 309-321). Berlin: Springer.

Cenoz, J., Gorter, D., \& May, S. (Eds.). (2017). Encyclopedia of language and education Language awareness and multilingualism. Berlin: Springer.

Chiss, J.-L. (1998). Plurivocité de l'écriture et "literacy.” Dans J.-G. Lapacherie (dir.), Propriétés de l'écriture (p. 89-94). Pau, France: Presses universitaires de Pau.

Chiss, J.-L. (2004). La littératie: quelques enjeux d'une réception dans le contexte éducatif et culturel français. Dans C. Barré-De Miniac, C. Brissaud et M. Rispail (dir.), La littératie: conceptions théoriques et pratiques d'enseignement de la lectureécriture (p. 43-52). Paris: L'Harmattan.

Chukly-Bonato, K. (2016). Transferring knowledge through translanguaging: The art of multilingualizing the foreign language classroom. (Unpublished master's thesis). Department of Integrated Studies in Education, McGill Uninversity, Montreal. digitool.library.mcgill.ca/R/-?func=dbin-jump-full\&current_base=GEN01\&object_id=143641 
Conseil de l'Europe. (2001). Cadre européen commun de référence: Apprendre, enseigner, évaluer. Strasbourg: Conseil de l'Europe. rm.coe.int/16802fc3a8

Conseil de l'Europe. (2018). Cadre européen commun de référence pour les langues: apprendre, enseigner, évaluer. Volume complémentaire avec de nouveaux descripteurs. Strasbourg: Conseil de l'Europe. rm.coe.int/cecr-volume-complementaireavec-de-nouveaux-descripteurs/16807875d5

Cook, G. (2010). Translation in language teaching. Oxford, England: Oxford University Press.

Creese, A., \& Blackledge, A. (2010). Translanguaging in the bilingual classroom: A pedagogy for learning and teaching? Modern Language Journal, 94, 103-115. doi.org/10.1111/j.1540-4781.2009.00986.x

Delcambre, I. (2012). De l'utilité de la notion de littéracies pour penser la lecture et l'écriture dans l'enseignement supérieur. Dans M.-C. Pollet (dir.), De la maîtrise du français aux littéracies dans l'enseignement supérieur (p. 19-35). Namur, Belgique: Presses universitaires de Namur.

Delisle, J. (2013). La traduction raisonnée: manuel d'initiation à la traduction professionnelle de l'anglais vers le français. (3e éd.). Ottawa, ON: Les Presses de l'Université d'Ottawa.

Dubuc, R. (2002). Manuel pratique de terminologie (4ème ed.). Brossard, QC: Linguatech.

Duverger, J. (2007). Didactiser l'alternance des langues en cours de DNL. Tréma, 28, 81-88. doi.org/10.4000/trema.302

Duverger, J. (2009). L'enseignement en classe bilingue. Paris: Hachette.

Eschenauer, S. (2014). Faire corps avec ses langues. Théâtre et didactique: vers une définition de la translangageance. E-CRINI, 6 . crini.univ-nantes.fr/actes-de-colloquelangues-en-mouvement-didactique-des-langues-et-pratiques-artistiques--1145716.kjsp

European Union. (2019, August). Interactive terminology for Europe (IATE) [v. 2.8.0]. iate.europa.eu/home

France. Ministère de la Culture. (n.d.). FranceTerme. www.culture.fr/franceterme

Gajo, L. et Steffen, G. (2015). Didactique du plurilinguisme et alternance de codes : le cas de l'enseignement bilingue précoce. Revue Canadienne des Langues Vivantes, 71, 471-499. doi.org/10.3138/cmlr.2740

García, O. (2009). Bilingual education in the 21st century: A global perspective. Malden, MA: Wiley-Blackwell.

García, O., Bartlett, L., \& Kleifgen, J. (2007). From biliteracy to pluriliteracies. In K. Knapp, D. Perrin, \& M. Verspoor (Series Eds.), Handbooks of applied linguistics [HAL]: Vol. 5, P. Auer \& L. Wei (Vol. Eds.), Handbook of multilingualism and multilingual communication (pp. 207-228). Berlin: Mouton de Gruyter.

García, O., \& Wei, L. (2014). Translanguaging: Language, bilingualism and education. Basingstoke, UK: Palgrave Macmillan. 
García, O., \& Lin, A.M. (2017). Translanguaging in bilingual education. In S. May (Series Ed.), Encyclopedia of language and education: Vol. 5, O. García, A. Lin, \& S. May (Vol. Eds.), Bilingual and multilingual education (pp. 117-130). Cham, Switzerland: Springer.

Gentil, G. (2002). Academic biliteracy and identity construction: Case studies of francophone science writers (Unpublished doctoral dissertation). Department of Integrated Studies in Education, McGill University, Montreal. digitool.library.mcgill.ca/ webclient/StreamGate?folder_id=0\&dvs=1571345681010 82\&usePid1 =true\&usePid2=true

Gentil, G. (2005). Commitments to academic biliteracy: Case studies of francophone university writers. Written Communication, 22, 421-471.

Gentil, G. (2006). EAP and technical writing without borders: The impact of departmentalization on the teaching and learning of academic writing in a first and second language. In P. Matsuda, C. Ortmeier-Hooper, \& X. You (Eds.), The politics of second language writing: In search of the promised land (pp. 147-167). West Lafayette, IN: Parlor Press.

Gentil, G. (2011). A biliteracy agenda for genre research. Journal of Second Language Writing, 20, 6-23. doi.org/10.1016/j.jslw.2010.12.006

Gentil, G. (2017). Afterword: Moving forward with academic biliteracy research. In D. Palfreyman \& C. van der Walt (Eds.), Academic biliteracies: Translanguaging and multilingual repertoires in higher education settings (pp. 206-220). Clevendon, UK: Multilingual Matters.

Gentil, G. (2018). Modern languages, bilingual education, and translation studies: The next frontiers in WAC/WID research and instruction? Across the Disciplines [thematic issue: Rewriting disciplines, Rewriting boundaries: Transdisciplinary and translingual challenges for WAC/WID], 15(3), 114-129. wac.colostate.edu/atd/special/ trans

Gentil, G. (2019). D'une langue à l'autre: pour une didactique plurilingue et translangagière de l'ècrit. Revue canadienne des langues modernes, 75, 65-83. doi.org/10.3138/cmlr.2018-0168

Gentil, G., \& Séror, J. (2014). Canada has two official languages - Or does it? Case studies of Canadian scholars' language choices and practices in disseminating knowledge. Journal of English for Academic Purposes, 13, 17-30. doi.org/10.1016/j.jeap.2013.10.005

Glorieux, C. (2015). Du concept de littéracie au champ des littéracies universitaires. Dans A. Belhadj Hacen et I. Delcambre (dir.), Littéracies et plurilinguismes: quelles pratiques? quels liens? (p. 37-59). Paris: L'Harmattan.

Göpferich, S. (2017). Cognitive functions of translation in L2 writing. In J.W. Schwieter \& A. Ferreira (Eds.), The handbook of translation and cognition (pp. 402-422). Hoboken, NJ: Wiley.

Guidère, M. (2016). Introduction à la traductologie. Louvain-la-Neuve, Belgium: De Boeck Supérieur. 
Halliday, M.A.K. (1978). Language as social semiotics. London: Edward Arnold.

Hébert, M., \& Lépine, M. (2013). De l'intérêt de la notion de littératie en francophonie : un état des lieux en sciences de l'éducation. On the relevance of the notion of literacy in Francophone countries: The situation in the field of education. Globe, 16(1), 25-43. doi.org/10.7202/1018176ar

Hornberger, N.H., \& Link, H. (2012). Translanguaging and transnational literacies in multilingual classrooms: a biliteracy lens. International Journal of Bilingual Education and Bilingualism, 15, 261-278. doi.org/10.1080/13670050.2012.658016

Hornberger, N.H., \& Skilton-Sylvester, E. (2003). Revisiting the continua of biliteracy: International and critical perspectives. In N.H. Hornberger (Ed.), Continua of biliteracy: An ecological framework for educational policy, research, and practice in multilingual settings (pp. 35-70). Clevedon, UK: Multilingual Matters.

Jaffré, J.-P. (2004). La litéracie: histoire d'un mot, effets d'un concept. Dans B.D. Miniac, C. Brissaud et M. Rispail (dir.), La littéracie: conceptions théoriques et pratiques d'enseignement de la lecture-écriture (p. 21-41). Paris: L'Harmattan.

John Benjamins. (2015). [On-line catalog description of Translation and Translanguaging in Multilingual Contexts]. benjamins.com/catalog/ttmc

Kruse, O. (2012). The place of writing in translation: From linguistic craftsmanship to multilingual text production. In C. Thais, G. Bräuer, P. Carlino, L. GanobcsikWilliams, \& A. Sinha (Eds.), Writing programs worldwide: Profiles of academic writing in many places (pp. 401-415). Fort Collins, CO: The WAC Clearinghouse. wac.colostate.edu/books/perspectives/wpww

Lewis, G., Jones, B., \& Baker, C. (2012). Translanguaging: Origins and development from school to street and beyond. Educational Research and Evaluation, 18, 641654. doi.org/10.1080/13803611.2012.718488

L'Homme, M.-C. (2008). Initiation à la traductique (2e éd.). Montréal, QC: Linguatech.

Liddicoat, T. et Zarate, G. (2009). La didactique des langues et des cultures face à la circulation internationale des idées. Le Français dans le Monde: Recherches et applications [numéro thématique: La circulation internationale des idées en didactique des langues], 46, 9-13.

Lillis, T.M., \& Curry, M.J. (2010). Academic writing in global context. New York: Routledge.

Loock, R. (2016). La traductologie de corpus. Villeneuve d'Ascq, France: Presses universitaires du Septentrion.

MacSwan, J. (2017). A multilingual perspective on translanguaging. American Educational Research Journal, 54, 167-201. doi.org/10.3102/0002831216683935

Maynard, C. et Armand, F. (2016). Écriture de textes identitaires plurilingues et rapport à l'écrit d'élèves immigrants allophones. Nouveaux cahiers de la recherche en éducation, 19, 134-160. 
Molinié, M. et Moore, D. (2012). Les littératies: une Notion en Questions en didactique des langues $(\mathrm{NeQ})$. Recherches en didactique des langues et des cultures: les Cahiers de l'Acedle, 9, 3-14. journals.openedition.org/rdlc/2757

Moore, D. (1996). Bouées transcodiques en situation immersive ou comment interagir avec deux langues quand on apprend une langue étrangère à l'école. Aile. Acquisition et interaction en langue étrangère, 7, 95-121. journals.openedition.org/aile/4912

Moore, D. (2001). Une didactique de l'alternance pour mieux apprendre? Ela. Études de linguistique appliquée, 121, 71-78.

Moore, D. (2006). Plurilinguismes et école. Paris: Didier.

Moore, D., \& Gajo, L. (2009). Introduction - French voices on plurilingualism and pluriculturalism: Theory, significance and perspectives. International Journal of Multilingualism [themtic issue: Plurilingualism and pluriculturalism: Francophone perspectives in education], 6, 137-227. doi.org/10.1080/14790710902846707

North, B. et Piccardo, E. (2016). Élaborer des descripteurs illustrant des aspects de la médiation pour le Cadre européen commun de référence pour les langues (CECR). Strasbourg: Conseil de l'Europe. mycloud.coe.int/index.php/s/VLAnKuMxDDsHK03

Otheguy, R., García, O., \& Reid, W. (2015). Clarifying translanguaging and deconstructing named languages: A perspective from linguistics. Applied Linguistics Review, 6, 281-307.

Painchaud, G. (1992). "Littératie” et didactique de l'écrit en L2. Études de linguistique appliquée, 88, 55-66.

Parini, L. (2010). Le concept de genre: constitution d'un champ d'analyse, controverses épistémologiques, linguistiques et politiques. Socio-logos, 5. journals.openedition.org/ socio-logos/2468

Pavlenko, A. (2005). Bilingualism and thought. In J.G. Kroll, A.M.B. de Groot (Eds.), Handbook of bilingualism: Psycholinguistic approaches (pp. 448-468). Oxford: Oxford University Press.

Pollet, M.-C. (2014). L'écrit scientifique à l'aune des littéracies universitaires : approches théoriques et pratiques. Namur, Belgique: Presses universitaires de Namur.

Quebec. Office québécoise de la langue française. (2012). Le grand dictionnaire terminologique (GDT). www.granddictionnaire.com/

Raus, R. (2013). La terminologie multilingue : la traduction des termes de l'égalité H/F dans le discours international. Bruxelles, Belgique: de Boeck.

Rehorick, S. (2011). The critical role of the educational system in creating bilingual citizens: New Brunswick as a microcosm of Canada. Ottawa, ON: Office of Commissioner of Official Languages. www.ocol.gc.ca/html/rehorick_e.php

Reuter, Y. (2003). La littératie. Perspectives pour la didactique. lidil. Revue de linguistique et de didactique des langues [numéro thématique: La littéracie: vers de nouvelles pistes de recherche didactique], 27, 11-23. 
Rispail, M. (2011). Littéracie: une notion entre didactique et sociolinguistique — enjeux sociaux et scientifiques. forumlecture.ch [numéro thématique: La littératie, un concept?], 1 . www.forumlecture. ch/archiv.cfm?issue $=1$ \&year $=2011$

Le Robert illustré. (2019). (Millésime 2019 éd.). Paris: Dictionnaires Le RobertSEJER.

Rughoonundun-Chellapermal, N. (2017). Autopsie de l'échec d'un projet d'éducation multilingue. Cahiers internationaux de sociolinguistique, 12, 155-177. doi.org/10.3917/cisl.1702.0155

Schrijver, I., van Vaerenbergh, L., Leijten, M., \& Van Waes, L. (2014). The translator as a writer: Measuring the effect of writing skills on the translation product. In D. Knorr, C. Heine, \& J. Engberg (Eds.), Methods in writing process research (pp. 99-121). Frankfurt, Germany: Peter Lang.

Schwinge, D. (2017). Biliteracy and multiliteracy in bilingual education. In J. Cenoz, D. Gorter, \& S. May (Eds.), Encyclopedia of language and education: Bilingual and multilingual education (pp. 147-160). Cham, Switzerland: Springer.

Swain, M. (2006). Languaging, agency and collaboration in advanced second language proficiency. In H. Byrnes (Ed.), Advanced language learning: The contribution of Halliday and Vygotsky (pp. 95-108). London: Continuum.

Thévenaz-Christen, T. (2011). Éditorial: La littératie, un concept? forumlecture.ch [numéro thématique: La littératie, un concept?], 1. www.forumlecture.ch/archiv.cfm? issue $=1$ \& year $=2011$

Trépos, J.-Y., Ehrhart, S., Hamez, G., Langinier, H., Polzin-Haumann, C. et Reissner, C. (2016). Frontières linguistiques et communautés de travail: un bilinguisme à l'épreuve du changement industriel. Questions de communications [numéro thématique: L'antibiorésistance, un problème en quête de publics], 16(29), 351-374.

Venuti, L. (2000). The translation studies reader. London: Routledge.

Vinay, J.-P. et Darbelnet, J. (1958). Stylistique comparée du français et de l'anglais. Montréal: Beauchemin.

Wei, L., \& García, O. (2014). Translanguaging: Language, bilingualism and education. New York: Palgrave Macmillan.

Wei, L., \& García, O. (2017). From researching translanguaging to translanguaging research. In J. Cenoz, D. Gorter, \& S. May (Eds.), Encyclopedia of language and education: Research methods in language and education (pp. 227-240). Cham, Switzerland: Springer.

Williams, C. (1994). Arfarniad o ddulliau dysgu ac addysgu yng nghyd-destun addysg uwchradd ddwyieithog [An evaluation of teaching and learning methods in the context of bilingual secondary education] (thèse de doctorat inédit). Bangor, UK: University of Wales.

Williams, C. (1996). Secondary education: Teaching in the bilingual situation. In C. Williams, G. Lewis, \& C. Baker (Eds.), The language policy: Taking stock: 
Interpreting and appraising Gwynedd's language policy in education (pp. 39-78). Llangefni, UK: Canolfan Astudiaethau Iaith (CAI) Language Studies Centre. 